\title{
Classification of Wild Isolate Nematode Strains Using Classical Systematics and Molecular Sequencing
}

\author{
Taylor Seacor ${ }^{\mathrm{a}}$, Miranda Striluk ${ }^{\mathrm{a}}$ and Carina Endres Howell ${ }^{\mathrm{a}}$
}

\begin{abstract}
Nematode worms account for the vast majority of the animals in the biosphere. They are critically important to public health and agriculture, reside in virtually all ecosystems, and are extremely diverse. To investigate nematode morphological diversity, three soil nematode species isolated in the wild where characterized (strains EM435, AF8130 and AF7340). These isolates were examined using differential interference contrast (DIC) microscopy, fluorescence microscopy and scanning electron microscopy (SEM). We used both classical systematics of the phenotypic data obtained from microscopy and phylogenetic evidence of ribosomal RNA sequencing data to classify these isolates. Data obtained confirmed strain EM435 to be a member of order Rhabditida, genus Oscheius, evidence was consistent with strain AF8130 belonging to the species Pristionchus pacificus, and the previously unclassified strain AF7340 was found to be a member of order Rhabditida, genus Oscheius.
\end{abstract}

Keywords: Nematode morphology; molecular phylogeny; scanning electron microscopy

\section{Introduction}

Nematodes are the most abundant multicellular organisms on earth (Platt, 1994). It is estimated that in addition to over 15,000 species of nematodes currently identified there are more than half a million species yet to be discovered (Gibbons, 2010). Nematodes are used as a model organism in the study of genetics, development and behavior, and pathogenesis (Fitch, 2005). In the field of evolutionary developmental biology, nematodes serve as important models for the understanding of genome diversity and conservation, cellular differentiation, morphogenesis and organogenesis. While nematodes are obviously different from mammals, discoveries over the past two decades have prompted a revolution in our understanding of the vast extent to which fundamental mechanisms of embryonic development (e.g. body patterning, gastrulation, early development of the nervous system, etc.) have been conserved widely across vertebrate and invertebrate phyla. Nematodes are also important to global public health as parasites, and to agriculture both as pathogens and as beneficial inhabitants of healthy soil.

In an effort to determine systematic nematode phylogeny, several methods have been implemented. Historically, nematodes have been classified through the study of morphological features using light microscopy. However, only a handful of systematic taxonomists have focused on nematode classifications by studying such features (De Ley, 2006). More commonly a holistic approach to nematode phylogeny was avoided because of the diversity of the ecology displayed by nematodes, and scientists favored specialization on one taxonomic group (Dorris, De Ley, \& Blaxter, 1999). In more recent years, molecular sequencing has come to predominate as a method of nematode classification with certain DNA sequences proving to be particularly reliable, such as the small subunit ribosomal RNA (SSU rRNA) genes.

Following the approach of De Ley and Blaxter (2002), the goal of this study was to integrate classical and molecular systematics to classify uncharacterized or partially characterized wild isolate strains of nematodes that were obtained from the Caenorhabditis Genetics Center (CGC). Three strains with varying levels of characterization were analyzed in total (see Table 1). The three experimental strains studied were EM435, AF8130 and AF7340. According to the CGC, Strain EM435 was isolated by David Fitch in Brooklyn, New York, United States in 1990 and has been partially sequenced but lacks morphological characterization. It has been positively identified as Oscheius myriophilia. AF8130 was isolated by Adras Fodor in Ontario, Canada and is thought to be Pristionchus pacificus due to successful matings, but has not been identified to a species level. AF8130 has also never been characterized morphologically or sequenced. AF7340 was also isolated by Andras Fodor in Ohio, United States and is an unknown species. It is an entirely uncharacterized strain on both morphological and molecular levels. A fourth strain, N2, was also analyzed but served as a control group, as it is positively identified as Caenorhabditis elegans. C. elegans was chosen as the control group due to its extensive characterization using both classical systematics and molecular sequencing (Haag, 2005).

Morphological characterizations were obtained using fluorescence microscopy, differential interference contrast (DIC) microscopy, and scanning electron microscopy (SEM). Amphid neurons were imaged for comparison using fluorescence microscopy. Specifically, a membrane-soluble fluorescence dye (DiI) was used to visualize chemosensory amphid neuron morphology in the various wild isolates. Amphid neurons are the anterior chemosensory neurons in nematodes, mediating critical behaviors including chemotaxis and mating. Lengths of nematodes and other anatomical features, such as vulva position, stylets, esophageal bulb, and tail terminus, were investigated using DIC microscopy. Buccal cavity (mouth) differences were observed and imaged using scanning electron microscopy to note minute variations in the jaw, amphid neurons, and the lips of the nematodes. SEM was also used to characterize cuticle annulation and other surface features of the nematodes. Molecular genome sequencing was used to determine molecular phylogenetic relationships within and between species. 18S Ribosomal 
RNA sequences were chosen for the molecular analysis. Combining characterization of both morphological traits and molecular sequence data allows for the determination of species specific information of the nematodes. Data obtained from these studies have allowed the classification of these three strains of nematodes.

\section{Results}

DIC Microscopy

DIC microscopy was used to compare major morphological characteristics of the different strains of nematodes. Like many, but not all, nematode species, $C$. elegans develops through four larval stages before the adult stage. Stage 4 larvae ("L4") of C. elegans, and the analogous pre-adult larvae of the other species, were picked for analysis. The standard criterion used for picking L4 stage larvae, namely identifying individuals with conspicuous vulval crescents appearing white under DIC optics, was used to pick presumably pre-adult terminal larval stage individuals from all species. Figure 1 shows representative full-length intact individuals from these apparently terminal larval stages from each species. There is considerable variance in length between strains (see Figure 1 and Table 2).

Table I: Strains of Nematodes classifies using microscopy and molecular techniques.

\begin{tabular}{ccc}
\hline Strain name & Place of origin & Collected by \\
\hline EM435 & Brooklyn, NY, USA & David Fitch \\
\hline AF8130 & Ontario, Canada & Adras Fodor \\
\hline AF7340 & Ohio, USA & Adras Fodor \\
\hline N2 (control) & Bristol, England & L.N. Staniland \\
& & \\
\hline
\end{tabular}

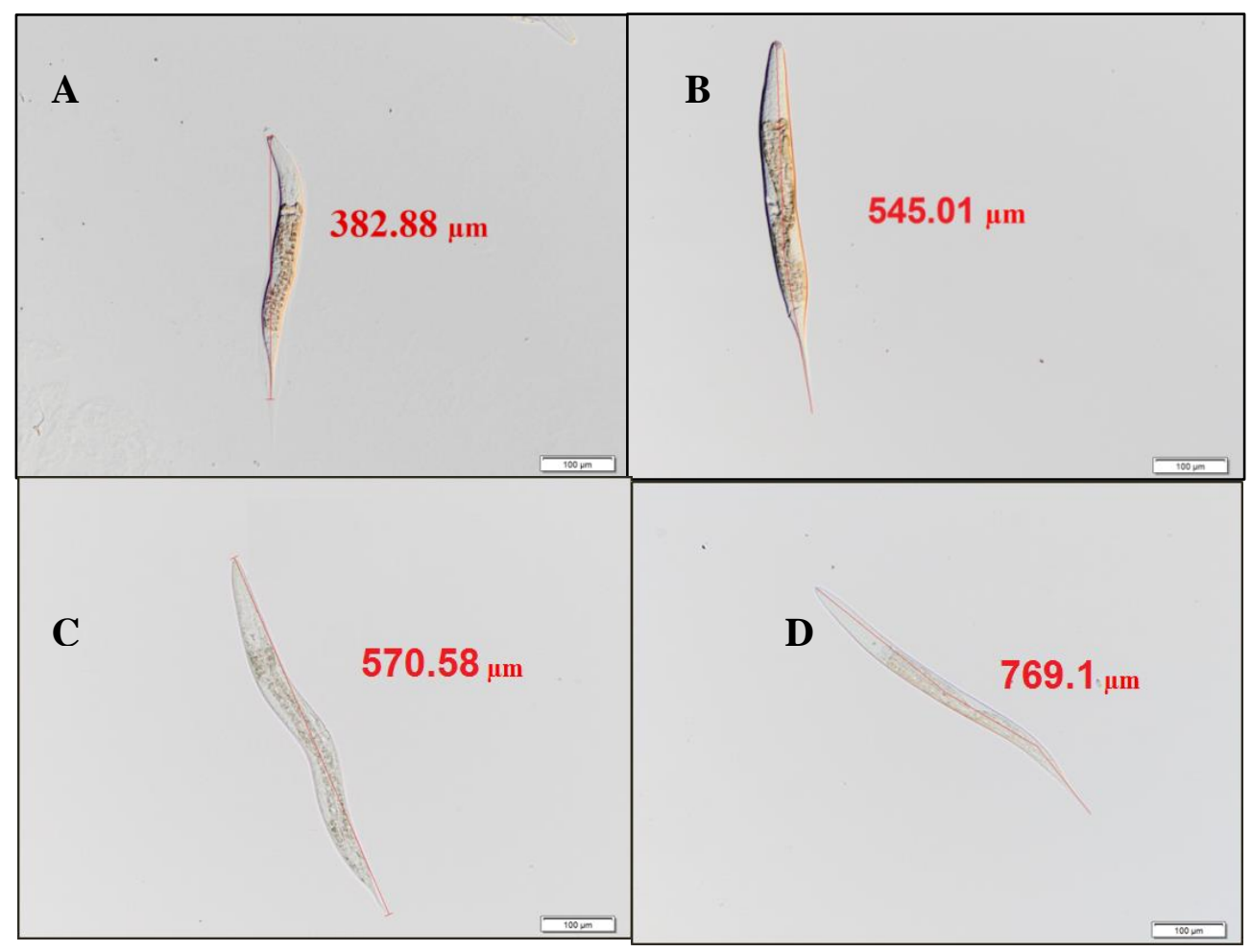

Figure 1: Full-length individual DIC microscopy images of nematode strains at terminal larval stage of development. Length of the body is indicated in microns $(\mu \mathrm{m})$. (A) AF7340, (B) AF8130, (C) EM435, (D) N2.

DIC microscopy was also used to compare the pharyngeal anatomy of the different strains of nematodes at the terminal larval stage (Figure 2). As can be seen in Figure $2, \mathrm{p}$ haryngeal length correlates with body length of each strain. The presence or absence of various anatomical characteristics was also observed in each strain, including stylet, teeth, basal bulb and median bulb. Results are shown in Table 2. 


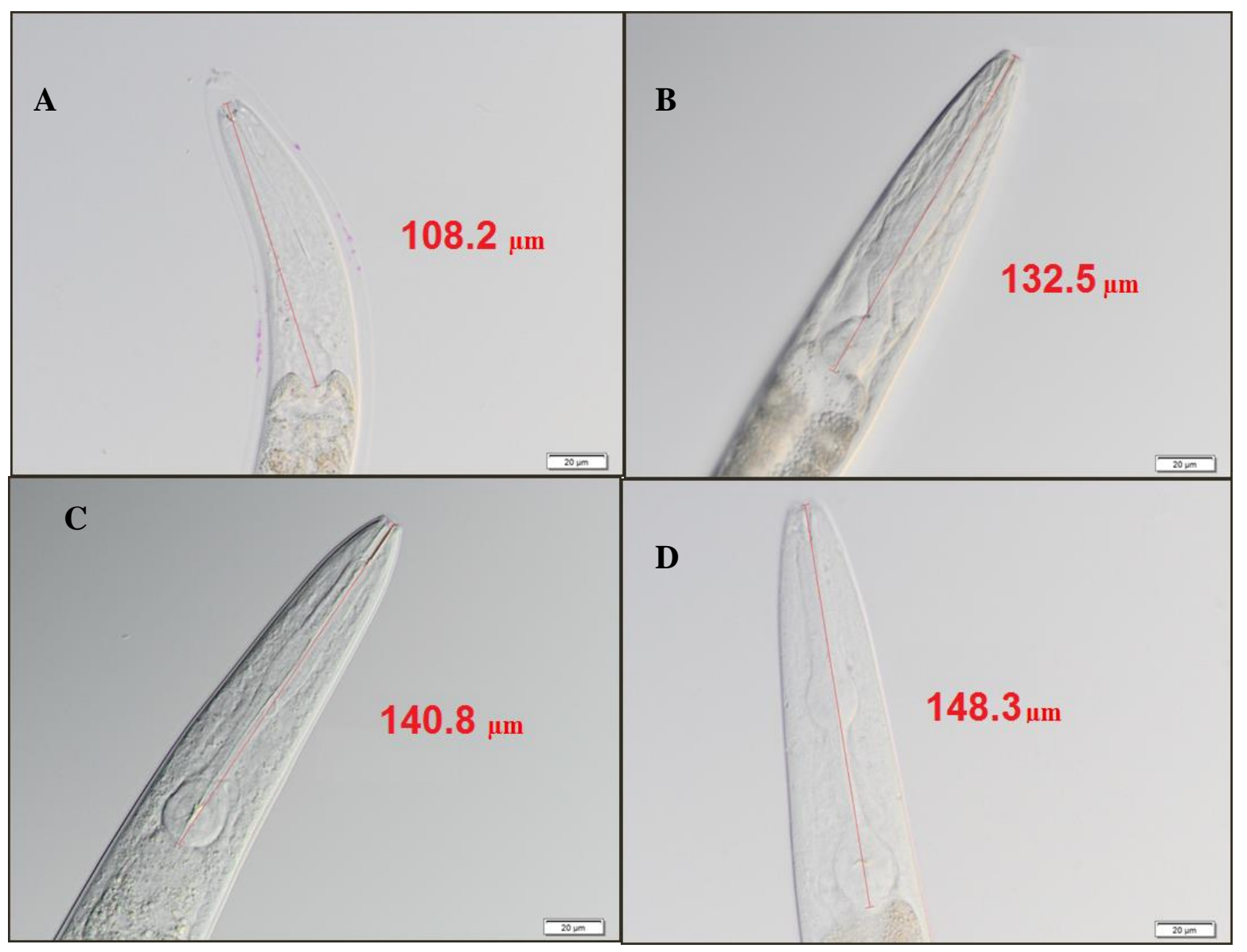

Figure 2: DIC images of the pharynx region of nematode strains at the terminal larval stage of development. Length of the pharynx is indicated in microns ( $\square \mathrm{m}$ ). (A) AF7340, (B) AF8130, (C) EM435, (D) N2.

\section{Fluorescence Microscopy}

In general, every cell in soil nematodes is separated from the environment by a non-permeable barrier called the cuticle. The single exception is the chemosensory dendrites of the anterior amphid neurons and posterior phasmid neurons which are contiguous with the environment through small passages in the cuticle. DiI stain was used to specifically label these neurons (Shaham, 2006) in the various wild isolates and the amphid morphology is described below.

Staining with DiI allowed visualization of the amphid neurons by specifically being taking up by the dendrites of the neurons in contact with the staining solution. The amphid neurons were successfully stained in all three strains, indicating that each strain did indeed have chemosensory amphid neurons whose dendrites had come into contact with the stain (Figure 3). Staining of the dendrites, cell bodies and axons that extend along the esophageal bulbs of the amphid neurons can be seen in each strain in Figure 3. Lengths of the axons were measured in each strain in microns (Table 2).

\section{Scanning Electron Microscopy (SEM)}

Scanning electron microscopy was used to investigate the buccal cavity differences and cuticle annulation among the nematode strains. Clear variations were present in the buccal cavities of different species (Figure 4). Morphology indicated adaptations to specific food sources. AF8130 showed pronounced teeth consistent with the predaceous tendencies of Pristionchus pacificus (Figure 4). AF7340 had a buccal cavity that resembled free living N2 and EM435 strains (Figure 4). Strains AF7340, AF8130 and N2 all had slight cuticle annulation, while EM435 had more moderate annulation as can be seen in Figure 4. These findings are summarized in Table 2. 
18S Small Subunit rRNA Sequencing

In order to characterize the molecular phylogeny of the strains, the $18 \mathrm{~S}$ small subunit ribosomal RNA (SSU rRNA) gene was chosen for sequencing analysis. This genetic region has proven to be useful for molecular phylogeny of nematodes in previous studies (Floyd R., 2002) (Floyd, R.M, 2005). The SSU rRNA gene DNA was successfully amplified using the Polymerase Chain Reaction (PCR) from strains AF7340 and EM435. PCR products were sequenced.

SSU rRNA sequence from the EM435 strain was analyzed using BLAST (Basic Local Alignment Search Tool) at the NCBI (National Center for Biotechnology Information) website to determine if genetic information has already been determined for identical or similar species. The EM435 stain SSU rRNA sequence showed $100 \%$ sequence identity with the Genbank entry AF082994 for Oscheius sp. (data not shown). SSU rRNA sequence from the AF7340 strain was also analyzed using BLAST. The AF7340 strain SSU rRNA sequence showed $100 \%$ sequence identity with the Genbank entry HQ130503 an Oscheius sp. (data not shown). The Oscheius genus falls under the order Rhabditida, which is consistent with the morphological observations.

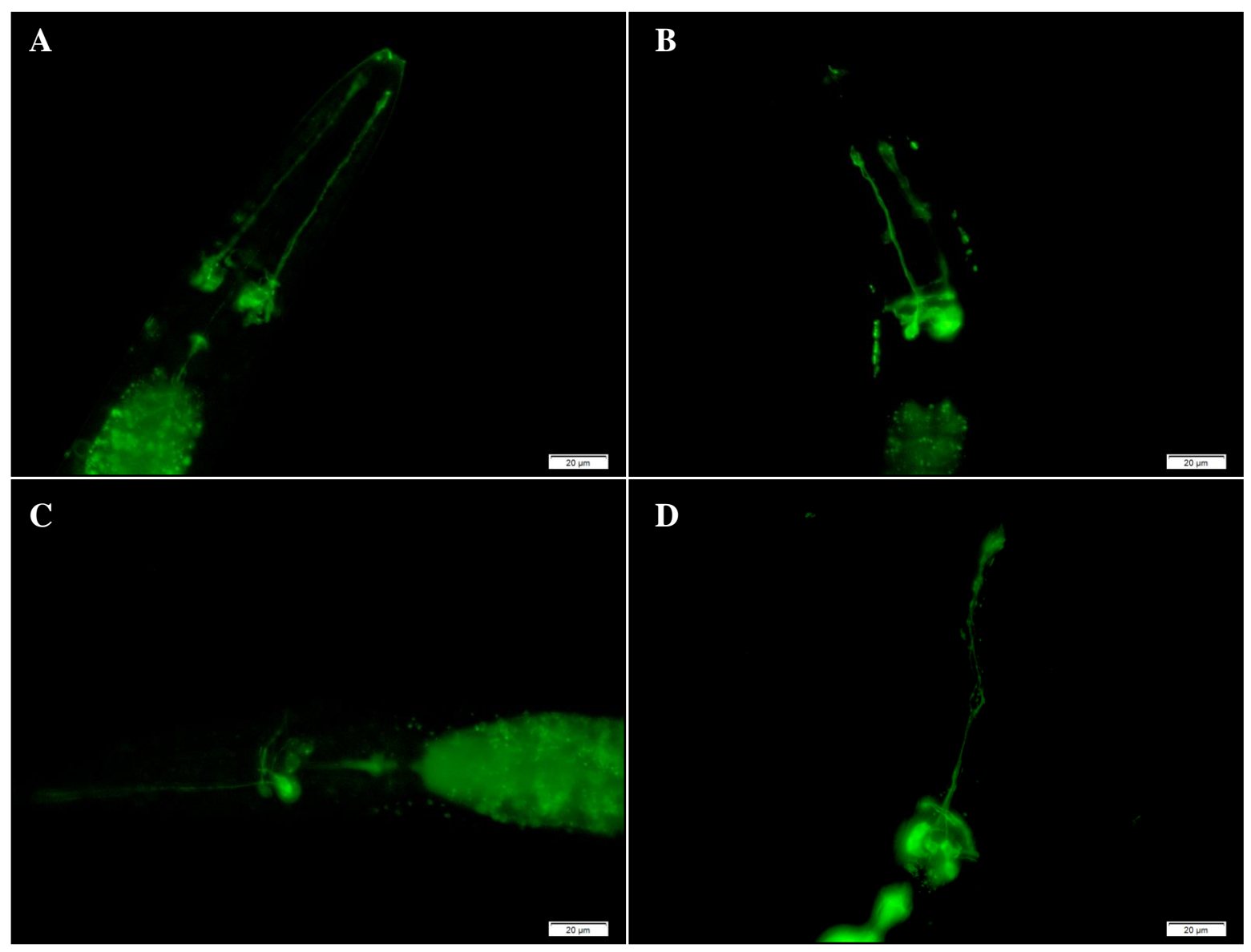

Figure 3: DiI stained amphid neurons. (A) AF7340, (B) AF8130, (C) EM435, (D) N2. 

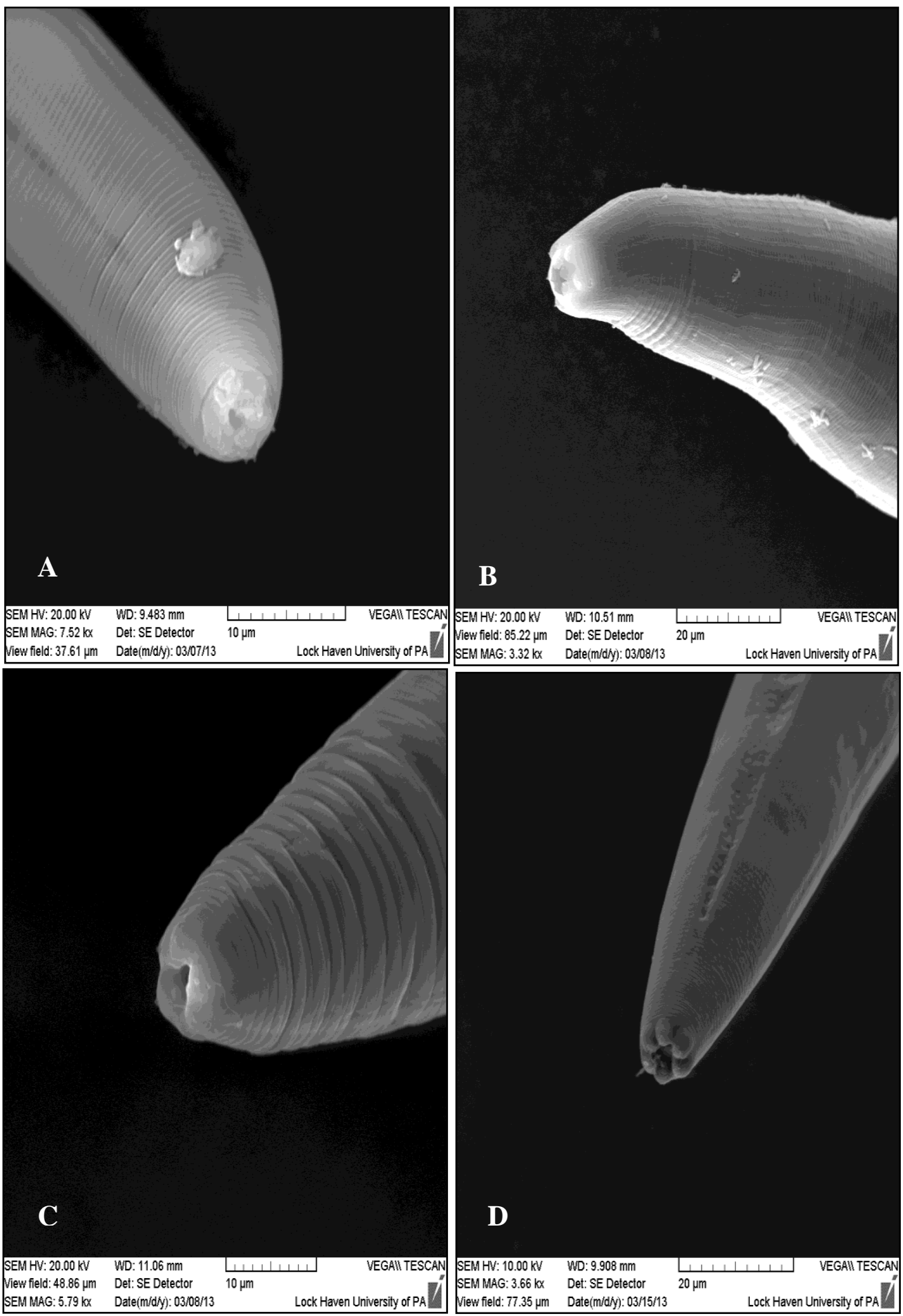

Figure 4: SEM images of buccal cavities and cuticle annulation among nematode strains. (A) AF7340, (B) AF8130, (C) EM435, (D) N2. 
Table II: Morphological data obtained using DIC microscopy, fluorescence microscopy, and scanning electron microscopy.

\begin{tabular}{|c|c|c|c|c|}
\hline Characteristic & AF7340 & AF8130 & EM435 & $\mathbf{N 2}$ \\
\hline Average Length $(\mu \mathrm{m})$ & $\begin{array}{l}484.26 \pm 74.2 \\
N=10\end{array}$ & $\begin{array}{l}602.35 \pm 92.5 \\
N=10\end{array}$ & $\begin{array}{l}568.89 \pm 113.4 \\
\mathrm{~N}=10\end{array}$ & $\begin{array}{l}820.1 \pm 201.0 \\
\mathrm{~N}=10\end{array}$ \\
\hline Vulva Position & Mid-body & Mid-body & Mid-body & Mid-body \\
\hline Stylet & Absent & Present & Absent & Absent \\
\hline Teeth & Absent & Present & Absent & Absent \\
\hline Basal Bulb & Prominent & Prominent & Prominent & Prominent \\
\hline Median Bulb & Absent & Absent & Present & Present \\
\hline Tail Terminus & Pointed & Pointed & Pointed & Pointed \\
\hline Annulation & Slight & Slight & Moderate & Slight \\
\hline Visible Amphid Neurons & Yes & Yes & Yes & Yes \\
\hline Axon Length $(\mu \mathrm{m})$ & 71.86 & 87.45 & 96.94 & 99.89 \\
\hline
\end{tabular}

\section{Discussion}

By characterizing nematode diversity and studying the development of wild isolate strains, one goal is to investigate similarities and differences in development between these distinct species and the well characterized $C$. elegans. By conducting comparative studies, we can elucidate common developmental mechanisms and differences between nematode species. The conservation of fundamental developmental mechanisms between $C$. elegans and humans at both the cellular and organ level can lead to a better understanding of the development of humans.

From our findings, strain AF8130 was confirmed to be correctly classified by genus using morphological data. EM435 sequence showed a $100 \%$ match to Oscheius sp. and was also supported by the morphological data as having this classification. Strain AF7340 was completely unclassified before this study, and was found to be a member of order Rhabditida, genus Oscheius as predicted by sequencing data and supported by the morphological data.

Future work will include a continuation of classical and molecular methods of analysis to characterize and classify additional nematode wild isolates, with the aim of finding unknown species for further investigation, including comparing and contrasting developmental mechanisms with those of $C$. elegans. Using both classical methods of microscopy and molecular methods to identify nematodes species is critical for a comprehensive comparison of the developmental mechanisms of new nematode species.

\section{Experimental Procedures}

\section{Strain Maintenance}

The four strains used for experimentation were obtained from the Caenorhabditis Genetics Center and maintained on Normal Growth Media (NGM) plates at 15 or $20^{\circ} \mathrm{C}$. Nematodes were propagated by a chunking process which involved using a sterile spatula and cutting a chunk of NGM agar plate $1 \mathrm{~cm} \times 1 \mathrm{~cm}$ on size, with a small number of nematodes on it, and then inverting the chunk and sliding it onto a fresh NGM plate spotted with E. coli strain OP50 as a food source (Stiernagle, 2006).

\section{Fluorescence Microscopy}

An NGM plate with the selected nematode strain was flooded with $1 \mathrm{~mL}$ of M9 solution (Stiernagle, 2006) and then decanted into a sterile eppendorf tube and centrifuged at 3000 $\mathrm{rev} / \mathrm{min}$. Carefully avoiding the nematode pellet, the supernatant was decanted off and the pellet was resuspended in $1 \mathrm{~mL}$ of $\mathrm{M} 9$ solution. $5 \mu \mathrm{L}$ of DiI stock solution (1:200 dilution of $2 \mathrm{mg} / \mathrm{ml}$ DiI [1; 1'-Dioctadecyl-3; 3; 3'; 3'tetramethylindocarbocyanine iodide, Fisher Scientific, Pittsburgh, PA] in dimethyl formamide) was added to eppendorf tube (Shaham, 2006). The tube was covered and left on a shaker for a minimum of 3 hours and a maximum of 24 hours. Nematodes were pelleted, washed with M9 $(2 \times 1 \mathrm{~mL})$, and transferred onto a microscope slide with an agarose pad and $10 \mu \mathrm{L}$ of Levamisol $\mathrm{HCl}$. Nematodes were imaged using an Olympus BX53 microscope using an ultraviolet light source. 


\section{Differential Interference Contrast (DIC) Microscopy}

Nematodes were picked individually from NGM plates and transferred onto a microscope slide with an agarose pad and $10 \mu \mathrm{L}$ of Levamisol $\mathrm{HCl}$. Around 4-6 nematodes were placed onto each slide. Nematodes were imaged using an Olympus BX53 microscope using a visible light source.

\section{Scanning Electron Microscopy (SEM)}

The primary fixation was conducted by immersing the nematodes in $2.5 \%$ glutaraldehyde and $0.1 \mathrm{M}$ phosphate buffer ( $\mathrm{pH}$ 7.4) for two hours at room temperature. Following the primary fixation, a primary wash was conducted $(3 \times 10$ minute intervals in $0.1 \mathrm{M}$ phosphate buffer). The secondary fixation proceeded by immersing the sample in $1 \%$ aqueous osmium tetroxide ( $\mathrm{pH}$ 7.4). The samples remained submerged in the $\mathrm{OsO}_{4}$ for one hour at room temperature in sealed containment within a fume hood. A secondary wash was conducted the same as the primary wash. Dehydration of the sample then followed using a series of graded ethanol solutions. Samples were let to sit until they formed a pellet and ethanol was then decanted.

Nematode strains were critical point dried in porous pots using an EMS850 Critical Point Dryer (Electron Microscopy Services, Hatfield, PA) with a chamber pre-cooled to $5{ }^{\circ} \mathrm{C}$. The chamber was filled with $\mathrm{CO}_{2}$ and temperature was kept below $5{ }^{\circ} \mathrm{C}$. To enhance solvent exchange, the stirrer was turned on for 5 minutes before the chamber was purged for 1 minute before a balanced flow of $\mathrm{CO}_{2}$ was introduced into the chamber. Once the heater to reach critical point: $37^{\circ} \mathrm{C}$ (pressure >1200 psi), the system was depressurized (100 $\mathrm{psi} / \mathrm{min}$ ) using the bleed valve.

Nematodes were mounted on SEM stubs using double sided carbon sticky tape. Roughly $200 \AA$ of gold was deposited on the sample surface using a Hummer VI sputter coater (Anatech USA, Union City, CA). The gold coating was applied over a 6 minute cycle (100 psi, 5mA). Samples were imaged at 10 and $20 \mathrm{HV}$ using a Vega II SEM (Tescan, Czech Republic).

\section{DNA Extractions}

Two different methods were employed to isolate DNA from nematode strains. Both extraction methods produced quality DNA that could be sequenced. The first method involved individually picking twenty nematodes of the same strain into $20 \mu \mathrm{L}$ of $0.25 \mathrm{M} \mathrm{NaOH}$. Samples were kept at room temperature for 3-16 hours before the lysate was heated for 3 minutes at $95{ }^{\circ} \mathrm{C} .4 \mu \mathrm{L} \mathrm{HCl}$ and $10 \mu \mathrm{L}$ of $0.5 \mathrm{M}$ Tris$\mathrm{HCl}(\mathrm{pH}$ 8) were added to neutralize. $5 \mu \mathrm{L} 2 \%$ Triton X-100 was added and the lysate was heated for 3 minutes at $95{ }^{\circ} \mathrm{C}$ (Floyd, 2002).

An alternate DNA extraction method was performed using the MoBio PowerSoil DNA Isolation Kit (MoBio, Carlsbad, CA). Five nematodes were placed into the MoBio bead tubes and the extraction was done following the manufacturer's protocol for bench to centrifuge extraction with the modification that mechanical agitation was done using a Disruptor Genie (Scientific Industries, Inc., Bohemia, New York) at $3000 \mathrm{rpm}$ for 10 minutes. Final volume eluted was $50 \mu 1$.

\section{Polymerase Chain Reaction (PCR)}

PCR was performed in duplicate on Genomic DNA samples extracted from nematodes (see above methods) using the Taq PCR Core kit (Qiagen, Valencia, CA). PCR reactions were a total volume of $40 \mu 1$ and performed in snap-cap PCR tubes. Each reaction was prepared with $1 \mathrm{X}$ buffer, $0.8 \mathrm{mM}$ $\mathrm{dNTP}, 0.025 \mathrm{u} / \mu \mathrm{l} \mathrm{Taq}$ polymerase, $3.0 \mathrm{mM} \mathrm{MgCl}_{2}, 0.5 \mu \mathrm{M}$ each of the forward and reverse primer, and $2 \mu \mathrm{L}$ of the target DNA. The final reaction volume was brought up to $40 \mu \mathrm{L}$ using sterilized distilled water. The $18 \mathrm{~S}$ rRNA gene was targeted using alternative two primer sets; Nem_18S_F primer (CGCGAATRGCTCATTACAACAGC) and Nem_18_R primer (GGGCGGTATCTGATCGCC) (Floyd, 2005) and SSU18A (AAAGATTAAGCCATGCATG) and SSU26R (AGCTGGAAT TACCGCG GCT G) (Blaxter, 1998). Amplification was carried out using a GeneAmp PCR System 9700 PCR machine (Applied Biosystems, Grand Island, NY) for 5 minutes at $94{ }^{\circ} \mathrm{C}$, [ 30 seconds $94{ }^{\circ} \mathrm{C}, 30$ seconds $54{ }^{\circ} \mathrm{C}, 1$ minute $72{ }^{\circ} \mathrm{C}$ ] repeated 35 cycles, and 10 minutes $72{ }^{\circ} \mathrm{C}$ (Floyd, 2005). Samples were then held at $4{ }^{\circ} \mathrm{C}$ or stored at $-20{ }^{\circ} \mathrm{C}$.

For each reaction, PCR products were visualized using a $3 \%$ agarose gel run with $10 \mu 1$ of each PCR reaction. In cases where 18S rRNA bands were present, PCR products were then cleaned using the QIAquick PCR Purification Kit (Qiagen, Valencia, CA), according to the manufacturer's protocol, and eluted to a final volume of $35 \mu 1$. The total concentrations and purity of the amplified DNA was determined by UV spectroscopy.

\section{DNA Sequencing}

Sequencing reactions were performed at the Pennsylvania State University's Core Genomics Facility using the Nem_18S_F, Nem_18_R, SSU18A or SSU18A primers using a 3730XL sequencer (Applied Biosystems, Grand Island, NY). Sequencing data was analyzed using BLAST at NCBI.

\section{Acknowledgements}

Support for this project was provided by National Science Foundation grants (DUE-1058829, DMR-0923047 and DUE0806660) to the Lock Haven University Nanotechnology program, as well as Lock Haven University of Pennsylvania Faculty Professional Development Committee grants to C. Howell. Thanks go to M. Tzolov and B. Overton for technical support of this work and A. Goonewardene for support of the Nanotechnology program, which was fundamental for this study to be completed. Some strains were provided by the CGC, which is funded by NIH Office of Research Infrastructure Programs (P40 OD010440). 


\section{References}

Barrière, A. and Félix, M.-A. (2006). Isolation of C. elegans and related nematodes. WormBook, ed. The C. elegans Research Community, WormBook, doi/10.1895/wormbook.1.115.1, http://www.wormbook.org.

Blaxter M.L., De Ley, P., Garey, J.R., Liu L.X., Scheldeman, P., Vierstraete, A., Vanfleteren, J.R., Mackey, L.Y., Dorris, M., Frisse, L.M., Vida, J.T., and Thomas, W.K. (1998). A molecular evolutionary framework for the phylum Nematoda. Nature, 392(6671), 71-75.

De Ley, P. (2006). A quick tour of nematode diversity and the backbone of nematode phylogeny. WormBook, ed. The C. elegans Research Community, WormBook, doi/10.1895/wormbook.1.41.1, http://www.wormbook.org.

Dorris, M., De Ley, P., and Blaxter, M. (1999). Molecular Analysis of Nematode Diversity and the Evolution of Parasitism. Trends in Parasitology, 15(5), 188-193.

Eisenback, J. D. (1986). A Comparison of Techniques Useful for Preparing Nematodes for Scanning Electron Microscopy. Journal of Nematology, 18(4), 479-487.

Fitch, D.H.A. (2005). Introduction to nematode evolution and ecology. WormBook, ed. The C. elegans Research Community, WormBook, doi/10.1895/wormbook.1.19.1, http://www.wormbook.org.

Floyd, R., Abebe, E., Papert, A., Blaxter, M. (2002). Molecular barcodes for soil nematode identification. Molecular Ecology , 11(4), 839-850.

Floyd, R.M., Rogers, A.D., Lambshead, P.J.D. and Smith, C.R. (2005). Nematode-specific PCR primers for the $18 \mathrm{~S}$ small subunit. Molecular Ecology Notes, 5, 611-612.

Gibbons, L. M. (2010). Keys to the Nematode Parasites of Vertebrates. Wallingford, Oxfordshire, UK: CAB International.

Haag, E. S. (2005). The evolution of nematode sex determination: C. elegans as a reference point for comparative biology. WormBook, ed. The C. elegans Research Community, WormBook, doi/10.1895/wormbook.1.120.1, http://www.wormbook.org.

Platt, H.M. (1994). Foreward. In: The phylogenetic systematics of free-living nematodes, S. Lorenzen, ed. (London: The Ray Society).

Shaham, S., ed., (2006). WormBook: Methods in Cell Biology, WormBook, ed. The C. elegans Research Community, WormBook, doi/10.1895/wormbook.1.49.1, http://www.wormbook.org

Stiernagle, T. (2006). Maintenance of C. elegans. WormBook, ed. The C. elegans Research Community, WormBook, doi/10.1895/wormbook.1.101.1, http://www.wormbook.org 